\title{
New times of minima and ephemeris for several OB eclipsing binaries
}

\author{
P. Mayer ${ }^{1}$, P.G. Niarchos ${ }^{2}$, R. Lorenz ${ }^{3}$, M. Wolf ${ }^{1}$, and G. Christie ${ }^{4}$ \\ 1 Astronomical Institute, Charles University, V Holešovičkách 2, 18000 Praha 8, Czech Republic \\ 2 Section of Astrophysics, Astronomy and Mechanics, Department of Physics, University of Athens, 15783 Zographos, Athens, \\ Greece \\ 3 Dr. Remeis-Sternwarte Bamberg, Astronomical Institute, University Erlangen-Nürnberg, Sternwartstraße 7, 96049 Bamberg, \\ Germany \\ 4 Astronomical Observatory, Auckland, New Zeeland
}

Received August 18; accepted December 12, 1997

\begin{abstract}
New times of minimum light were measured for several early-type eclipsing binaries: V337 Aql, V1182 Aql, V1331 Aql, IU Aur, QZ Car and V382 Cyg. The O-C diagram for these systems is discussed and new ephemeris is given for $\mathrm{AH}$ Cep; observation of $\mathrm{AQ}$ Cir is added. It is shown that the scatter of times of minima is several times larger than the measuring errors for most of the binaries studied.
\end{abstract}

Key words: stars: binaries: eclipsing — stars: early type

\section{Introduction}

The behaviour of orbital periods of early-type binaries is still very little known; in some cases, perhaps shallow minima or long periods make these variables not much attractive for observers. Nevertheless, interesting results were obtained for this type of binaries - e.g., in several cases, light-time effect due to a third body, or variations of $\mathrm{O}-\mathrm{C}$ values significantly larger than measuring errors were found. Some of the present authors have already discussed the subject (Mayer 1980, 1987; Mayer et al. 1991, hereafter MWTN). In this note we give newly measured times of minima for six variables and also discuss the periods in cases where more data are available. The stars selected are those with a longer observing history among the OB eclipsing binaries; they are also included in our program to determine absolute dimensions and evolutionary properties of such systems. The only exception, AQ Cir, is a candidate to be included in the program.

Send offprint requests to: P. Mayer

\section{Measurements}

The times of minima were measured at several observatories:

- Kryonerion Astronomical Station of the National Observatory of Athens, Greece - $1.2 \mathrm{~m}$ telescope,

- Carter Observatory, New Zeeland - 0.6 m telescope,

- Ondřejov Observatory of the Czech Academy of Science, Czech Republic - 0.65 m telescope,

- ESO La Silla Observatory, Chile - 0.5 m ESO telescope, and

- Wendelstein Observatory, University of Munich, Germany - $0.8 \mathrm{~m}$ telescope.

The telescope in Ondřejov has been equipped with a CCD camera, the other telescopes with photometers with photomultipliers. The newly measured minima are given in Table 1. Their times were determined by the Kwee-van Woerden (1956) method. Other published times of minima have been used in constructing the $\mathrm{O}-\mathrm{C}$ graphs, and their table can be obtained by e-mail from PM (mayer@mbox.cesnet.cz). In all cases where primary and secondary minima were measured or published, the phase of secondary minima is unrecognizable from 0.5 , i.e., the systems possess circular orbits.

\section{1. $V 337 \mathrm{Aql}$}

The older photoelectric minima were collected by Mayer (1987). It appears that a constant period suits all photoelectric minima. Since the minimum time given in Table 1 is the one with highest accuracy, we take it as the base for new ephemeris:

Pri.Min. $=$ HJD $2448779.5175+2$ d. $7338794( \pm 14) \cdot E$. 
Table 1. Observed times of minima

\begin{tabular}{lllcccl}
\hline Variable & HJD-2400000 & m.e. & Epoch & $\mathrm{O}-\mathrm{C}$ & $(\mathrm{O}-\mathrm{C})_{1}$ & Source \\
\hline V337 Aql & 48779.5175 & .0009 & 0 & .0000 & & Kryonerion \\
V1182 Aql & 48753.7520 & .0010 & 1533 & -.0035 & & La Silla \\
& 49158.4158 & .0007 & 1782.5 & -.0005 & & Kryonerion \\
& 49518.4752 & .0009 & 2004.5 & .0000 & & Kryonerion \\
& 49540.3678 & .0007 & 2018 & -.0029 & & Kryonerion \\
& 49600.3831 & .0006 & 2055 & .0026 & & Kryonerion \\
V1331 Aql & 48814.4219 & .0003 & 4548 & .0036 & & Kryonerion \\
& 49524.4757 & .0005 & 5068.5 & -.0063 & & Kryonerion \\
IU Aur & 48526.5392 & .0014 & 5563.5 & -.0048 & $.800^{a}$ & Kryonerion \\
& 49403.2920 & .0010 & 6047.5 & -.0059 & $.779^{a}$ & Ondřejov \\
QZ Car & 49422.039 & .002 & 0 & & & Mount John \\
V382 Cyg & 48839.6629 & .0003 & 6377.5 & .0143 & -.0036 & Wendelstein \\
& 48878.3252 & .0005 & 6398 & .0235 & .0053 & Wendelstein \\
& 49913.4785 & .0010 & 6947 & .0285 & .0019 & Ondřejov \\
\hline
\end{tabular}

${ }^{a}$ phase in the third-body orbit.

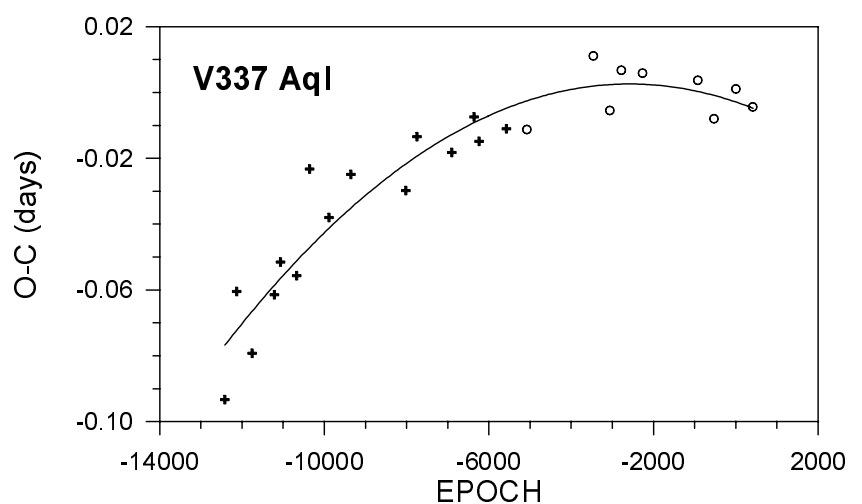

Fig. 1. O-C graph for V337 Aql; plus signs denote photographic data, circles photoelectric data

Note that rather unrealistic values for the orbital period are given in GCVS (2.733849) and SAC (2. 733826).

When older photographic minima are considered, it appears that the period continues to shorten, see Fig. 1. The reason of this shortening is unknown. Since light-time effect could be responsible for it, we took several coudé CCD spectra of this binary with the $2.2 \mathrm{~m}$ telescope of the German-Spanish Observatory at Calar Alto, in order to find spectroscopic hints for the existence of a third body. However, no third lines were found, i.e., the luminosity of the eventual third body cannot be larger than about $5 \%$ of the integral light of the system in the blue spectral region. From the deviations of the $\mathrm{O}-\mathrm{C}$ data from linearity the minimum mass of the eventual third body might be roughly estimated as about $1.5 M_{\odot}$; the expected luminosity of such a body is of course well under sensitivity of any present spectroscopy.

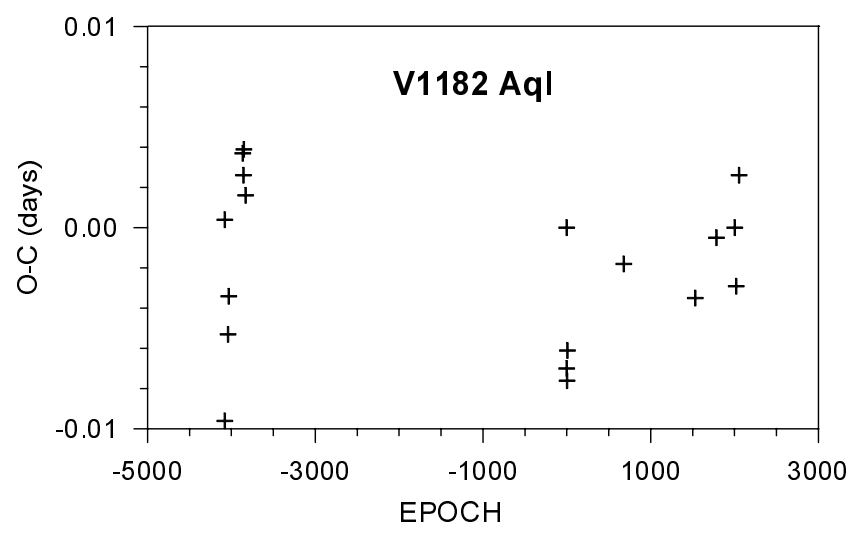

Fig. 2. O-C diagram of V1182 Aql

\section{2. $V 1182 \mathrm{Aql}$}

The $\mathrm{O}-\mathrm{C}$ column is calculated with the ephemeris by Bell et al. (1987):

Pri.Min. $=$ HJD $2446267.4027+1$ d $621887 \cdot E$.

The scatter of published times of minima is large, see Fig. 2. In this case lines of a third body were found on spectra taken by RL with the coudé auxilliary telescope feeding the $3.6 \mathrm{~m}$ telescope coudé spectrograph at ESO La Silla, and with the Calar Alto $2.2 \mathrm{~m}$ telescope and its coudé spectrograph. This system will be thoroughly discussed in a forthcoming paper by Lorenz et al. However, the fast changes of $\mathrm{O}-\mathrm{C}$ values are inexplicable by a lighttime effect. In Sect. 3 it is suggested that these changes might be connected with stability of the light curve. 


\subsection{V1331 Aql}

V1331 Aql is a detached early B-type binary, for which period and minimum times were published by Lorenz et al. (1991). The $\mathrm{O}-\mathrm{C}$ values presented in Table 1 were calculated using the ephemeris given in that paper:

Pri.Min. = HJD $2442610.0581+1.3641953 \cdot E$.

$U B V$ light curves were published by Lorenz et al. (1990). A detailed study of this system by Lorenz et al., including absolute dimensions derived from a recently established radial velocity curve and the light curve analysis, is in preparation.

\subsection{IU Aur}

New times of minima of this well-studied three-body system (Drechsel et al. 1994) should precise the period of the third body; theory also asks for a long-period (335 years) change of the orbital period (Mayer 1983). O-C values were calculated according to the ephemeris

Pri.Min. = HJD $2438448.4068+1$ d $81147435 \cdot E$

and phases in the third-body orbit according to

Phase $=($ HJD -2438579$) / 294.3$

(see Mayer 1987). Two minima given in Table 1 fit the older values of $\mathrm{O}-\mathrm{C}$ (and the theoretical curve given in Fig. 3 of Mayer 1990) very well, so no changes of periods in the orbit of the eclipsing pair or in the orbit of the third body are apparent.

\subsection{QZ Car}

This eclipsing variable is a part of a multiple system (see e.g. Morrison \& Conti 1980). The period of this variable is very close to 6 days, which means that minima can be measured well only in a limited interval of geographic longitudes in a given year. The observations published by now do not comprise any detailed measurements of the deepest part of a minimum of the QZ Car light curve. In Fig. 3 such measurements obtained at the Carter Observatory are plotted. These measurements were reduced using HD 93131 (a WR star, with $V=6.50, B-V=-0.03$ and $U-B=-0.88$ ) as the comparison star. This star, used as the comparison star also in the discovery paper by Walker \& Marino (1972) however might be slightly variable, the variability being of the order of 0.01 ; note that Morrison \& Conti recommend HD 93502. The time of the minimum calculated from these measurements is the most precise time known by now, so a new ephemeris is suggested (the period is according to Mayer et al. 1992):

Pri.Min. = HJD $2449422.039+5$ d.99857 $\cdot E$.

In the first part of the year 1998, the minima of QZ Car will be observable in South America.

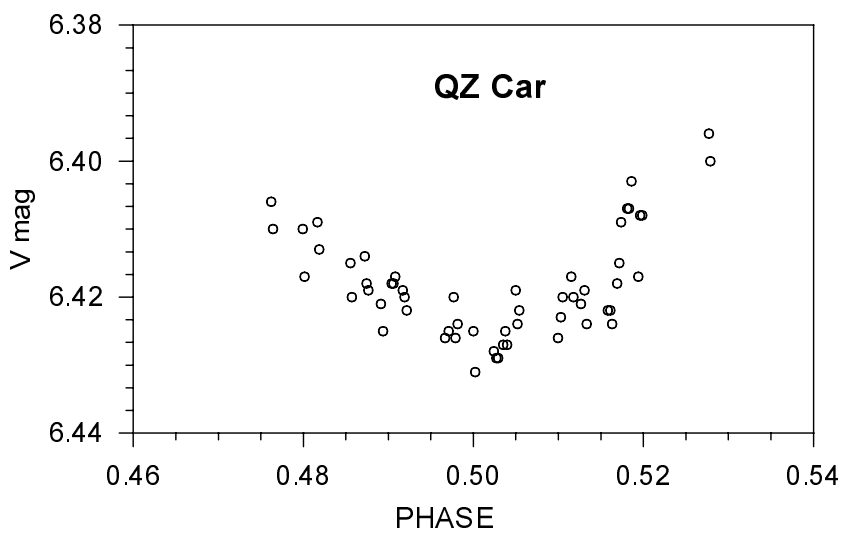

Fig. 3. $V$ magnitudes for QZ Car during a primary minimum

Table 2. Parameters of the light-time effect in the AH Cep system

\begin{tabular}{lcc}
\hline Parameter & Old value $^{a}$ & New value \\
\hline Long period & $62.9 \mathrm{yr}$ & $64.1 \mathrm{yr}$ \\
Semi-amplitude $A$ & 0.0683 & 0.059 \\
Eccentricity $e$ & 0.46 & 0.51 \\
Length of periastron $\omega$ & $122^{\circ}$ & $96^{\circ}$ \\
Time of periastron & JD 2445950 & JD 2444800 \\
$f\left(M_{3}\right)$ & $0.458 M_{\odot}$ & $0.261 M_{\odot}$ \\
Short period & $1^{\mathrm{d}} .7747420$ & $1^{\mathrm{d}} .7747433$ \\
HJD $_{0}$ & 2434989.455 & 2434989.459 \\
\hline
\end{tabular}

${ }^{a}$ according to Drechsel et al. (1989).

\section{6. $A H C e p$}

This bright detached eclipsing O-type binary, which is of special interest because of the pronounced light-time effect it displays, was thoroughly discussed by Drechsel et al. (1989). Here, 10 new minima published by other authors, which deviate systematically from the light-time effect curve as given in Fig. 3 of that study, were used to calculate a new set of the light-time effect parameters (see Table 2). The light-time effect curve corresponding to these parameters is shown in Fig. 4. The minima observed after $E=6000$ approximately follow a linear ephemeris Pri.Min. = HJD $2434989.676+1.774715 \cdot E$;

this ephemeris can be used to forecast minimum times in several next years.

\subsection{AQ Cir}

Except for the discovery paper (Hoffmeister 1943), this star was never studied. In GCVS the spectral type is given as OB, however, the source of this classification is unknown. There is a star No. 3268 in the catalogue Luminous Southern Stars (Stephenson \& Sanduleak 1971), with type $\mathrm{OB}(+) \mathrm{h}$, only 3 arcmin away; we used this star as the comparison star. GSC numbers, J2000 coordinates and magnitudes of both stars are: 


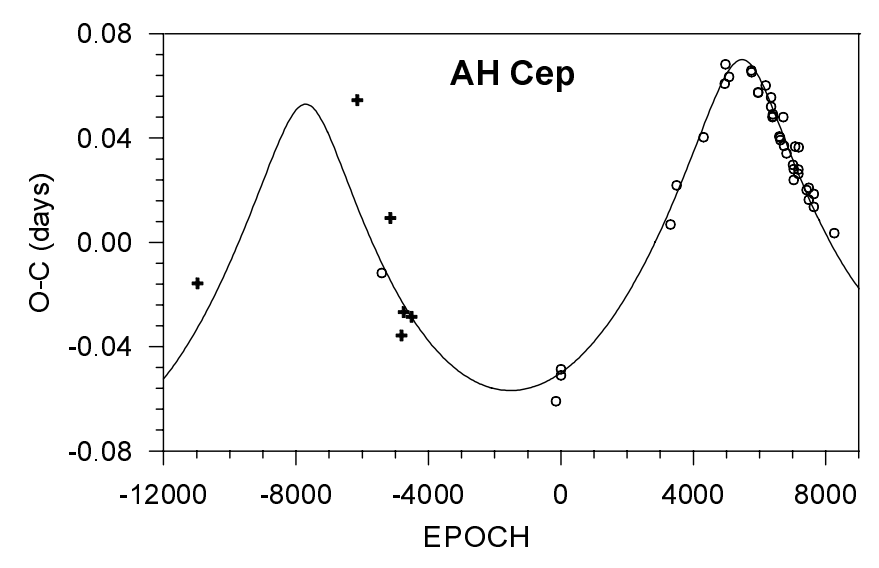

Fig. 4. O-C diagram for AH Cep. The curve corresponds to the light-time effect described by parameters given in Table 2; plus signs are photographic, circles photoelectric data

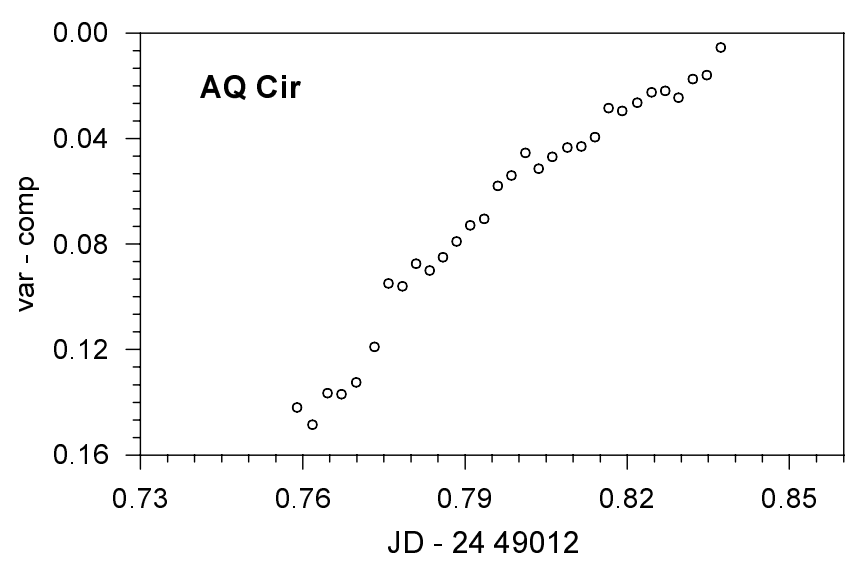

Fig. 5. AQ Cir, measurements in $B$ filter

$$
\begin{aligned}
& \text { AQ Cir: } \quad \text { GSC 9015.0071, CPD -64 } 2941 \\
& 14^{\mathrm{h}} 37^{\mathrm{m}} 19.89^{s} \quad-64^{\circ} 45^{\prime} 23^{\prime \prime} .8 \quad 11^{\mathrm{m}} 57
\end{aligned}
$$

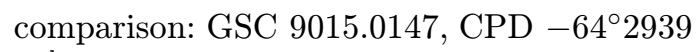

$$
\begin{aligned}
& 14^{\mathrm{h}} 37^{\mathrm{m}} 10.20^{\mathrm{s}} \quad-64^{\circ} 48^{\prime} 04^{\prime \prime} 3 \quad 10^{\mathrm{m}} \cdot 74 \text {. }
\end{aligned}
$$

Hoffmeister gives 7 times of minimum light and period 0.57284 (as he remarks, the true period is probably twice as long). From his original data it appears that the accuracy of the period is about \pm 0.00002 ; this means that at present, the epoch number is not known unambiguously. The large magnitude difference between the variable and comparison given in GSC means that in the time of exposition (JD 2446940.58) the variable had to be close to a minimum. We measured a part of the light curve covering a phase range after a minimum, and one can estimate when the minimum had appeared - perhaps at about HJD 2449012.67 \pm .05 . Unfortunately, no certain conclusion concerning the period can be reached, and further measurements are necessary. We present our measurements in Fig. 5 in order to facilitate the determination of

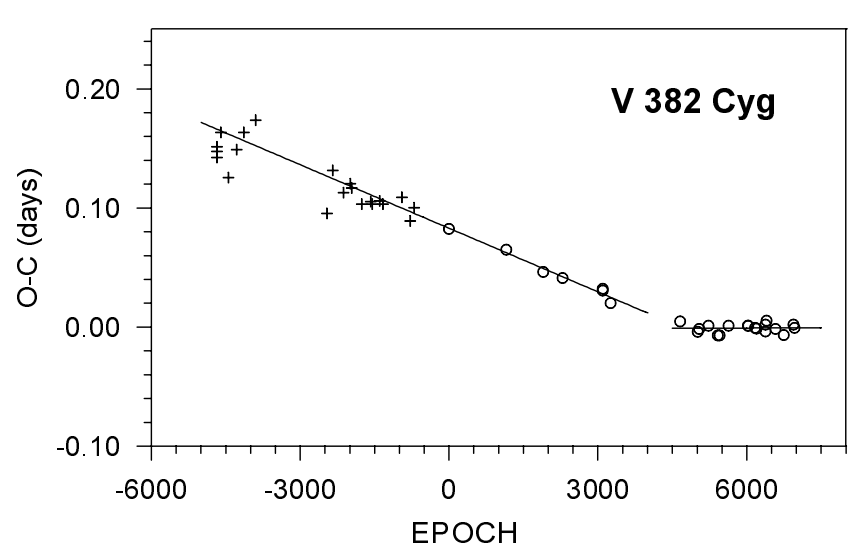

Fig. 6. V382 Cyg, $(\mathrm{O}-\mathrm{C})_{1}$ diagram. Plus signs are photographic, circles photoelectric data

the period (note that the GSC magnitudes correspond to $V$ magnitudes; we measured $B$ values).

\subsection{V382 Cyg}

Older minima of V382 Cyg observed before the year 1990 are listed by Mayer (1980) and MWTN. Several times of minima were published recently, namely by Agerer (1991, 1992, 1993, 1994, 1996) and by Agerer \& Hubscher (1995); we have also measured three more. The column $\mathrm{O}-\mathrm{C}$ is calculated with ephemeris by Mayer et al. (1986):

Pri.Min. = HJD $2436814.7703+1$ d $885516 \cdot E$.

During the time interval covered by observations the period has considerably lengthened (Mayer 1980). However, it appears that the period changed last around $E=4700$, but then it has been constant, so the presently valid ephemeris can be written as

Pri.Min. = HJD $2436814.680( \pm 8)+1.8855328( \pm 14) \cdot E$.

According to this ephemeris the column $(\mathrm{O}-\mathrm{C})_{1}$ of Table 1 has been calculated and Fig. 6 drawn. The photoelectric times of minima in the interval of epochs from 0 to 4700 fit also a linear ephemeris:

Pri.Min. = HJD $2436814.772( \pm 3)+1.8855143( \pm 9) \cdot E$.

\section{Discussion}

For most of the binaries discussed here, the presented data provide a significant improvement of the ephemeris. In case of AQ Cir, our observations might be helpful in findig an accurate value for the orbital period. Moreover, the collected data allow for a discussion of the scatter of times of minima. Our results suggest that for some binaries, this scatter is considerably larger than are the errors of measurements, whereas for other systems the scatter is comparable to these errors. We calculated the scatter of 
minimum times around the linear parts of ephemeris; the scatter is large for all binaries where such calculation was possible: V337, V1182, V1331 Aql and V382 Cyg. The scatter is large for $\mathrm{AH}$ Cep too (calculated after the effect of the third body had been removed). The rms values rise from 0.0033 for V382 Cyg to 0.0071 for V1331 Aql. IU Aur is known for a scatter of minimum times ( 0.0012 , after the effect of the third body is subtracted) only a little larger than are the measuring errors, and such a behaviour is present also for V606 Cen (scatter 0.00045, average m.e. 0 d00032) studied in a paper by Lorenz et al. (in preparation).

According to other data it seems that for all five binaries with large scatter, the light curve is intrinsically variable, in some cases (V337 Aql) it is also asymmetric.

\section{References}

Agerer F., 1991, BAV Mitteilungen 59 Agerer F., 1992, BAV Mitteilungen 60 Agerer F., 1993, BAV Mitteilungen 62 Agerer F., 1994, BAV Mitteilungen 68
Agerer F., 1996, IBVS 4383

Agerer F., Hübscher, 1995, IBVS

Bell S.A., Hilditch R.W., Adamson A.J., 1987, MNRAS 225, 961

Drechsel H., Haas S., Lorenz R., Mayer P., 1994, A\&A 284, 863

Drechsel H., Lorenz R., Mayer P., 1989, A\&A 221, 49

Hoffmeister C., 1943, Kleine Veröf. Berlin-Babelsberg 27

Kwee K.K., van Woerden H., 1956, BAN 12, 327

Lorenz R., Mayer P., Drechsel H., 1990, IBVS 3599

Lorenz R., Drechsel H., Mayer P., 1991, IBVS 3629

Mayer P., 1980, BAC 31, 292

Mayer P., 1983, BAC 34, 335

Mayer P., 1987, BAC 38, 58

Mayer P., 1990, BAC 41, 231

Mayer P., Wolf M., Muminović M., Stupar M., 1986, IBVS 2942

Mayer P., Wolf M., Tremko J., Niarchos P., 1991, BAC 42, 225

Mayer P., Drechsel H., Lorenz R., 1992, IBVS 3805

Morrison N., Conti P., 1980, ApJ 239, 212

Stephenson C.B., Sanduleak N., 1971, Publ. Warner and Swasey Obs. 1, 1

Walker W.S.G., Marino B.F., 1972, IBVS 681 